\title{
Bioengineered lungs generated from human iPSCs-derived epithelial cells on native extracellular matrix
}

\author{
Mahboobe Ghaedi $^{1,2}$ (1) | Andrew V. Le ${ }^{1,2}$ (D) | Go Hatachi ${ }^{1,2}$ | Arkadi Beloiartsev ${ }^{1,2}$ । \\ Kevin Rocco ${ }^{1,2} \mid$ Amogh Sivarapatna ${ }^{1,2}$ | Julio J. Mendez ${ }^{1,2}$ | Pavlina Baevova ${ }^{1,2}$ | \\ Rachel N. Dyal ${ }^{3}$ | Katie L. Leiby ${ }^{1,2}$ | Eric S. White ${ }^{3}$ | Laura E. Niklason ${ }^{1,2}$
}

${ }^{1}$ Department of Anesthesiology, Yale University, New Haven, CT, USA

${ }^{2}$ Department of Biomedical Engineering, Yale University, New Haven, CT, USA

${ }^{3}$ Internal Medicine, Pulmonary and Critical Care, University of Michigan, Ann Arbor, MI, USA

\section{Correspondence}

Laura E. Niklason, Departments of Anesthesia and Biomedical Engineering, Yale University,

New Haven, CT 06520, USA.

Email: laura.niklason@yale.edu

Funding information

National Institute of Health (NIH), Grant/

Award Number: U01HI110967, R01

HL098220, U01 HL111016 and DK65988

\begin{abstract}
The development of an alternative source for donor lungs would change the paradigm of lung transplantation. Recent studies have demonstrated the potential feasibility of using decellularized lungs as scaffolds for lung tissue regeneration and subsequent implantation. However, finding a reliable cell source and the ability to scale up for recellularization of the lung scaffold still remain significant challenges. To explore the possibility of regeneration of human lung tissue from stem cells in vitro, populations of lung progenitor cells were generated from human iPSCs. To explore the feasibility of producing engineered lungs from stem cells, we repopulated decellularized human lung and rat lungs with iPSC-derived epithelial progenitor cells. The iPSCs-derived epithelial progenitor cells lined the decellularized human lung and expressed most of the epithelial markers when were cultured in a lung bioreactor system. In decellularized rat lungs, these human-derived cells attach and proliferate in a manner similar to what was observed in the decellularized human lung. Our results suggest that repopulation of lung matrix with iPSC-derived lung epithelial cells may be a viable strategy for human lung regeneration and represents an important early step toward translation of this technology.
\end{abstract}

KEYWORDS

decellularized lung, epithelial cells, induced pluripotent stem cell (iPSC), lung implant, tissue regeneration bioreactor

\section{1 | INTRODUCTION}

Engineered lungs made using decellularized allogeneic or xenogeneic lungs and repopulated with patient-specific cells may be a future option for the replacement of diseased lungs (Badylak, Weiss, Caplan, \& Macchiarini, 2012; McCurry et al., 2009; Price, England, Matson, Blazar, \& Panoskaltsis-Mortari, 2010; Song \& Ott, 2011; Tesei et al., 2009; Wagner et al., 2013). Lung tissues that provide gas exchange have been generated from decellularized scaffolds and primary epithelial cells and successfully transplanted into small animal models for periods of a few hours (Ott et al., 2010; Petersen et al., 2010).

A key hurdle for the reconstruction of human lung tissue is the lack of an abundant source of human lung epithelial cells (Brouwer, Hoogenkamp, Daamen \& van Kuppevelt, 2013; Gilpin, Charest, Ren, \& Ott, 2016; Petersen, Calle, \& Niklason, 2011; Rana, Anson, Engle, \& Will,
2012; Wagner et al., 2013). Primary cells isolated from patients can rapidly lose phenotypic marker expression in vitro, and isolation of large numbers of cells for expansion is difficult and costly. This may limit the application of these primary cells for lung tissue engineering. Human induced pluripotent stem cells (iPSCs) are a potentially attractive source for the generation of lung epithelial cells, though differentiation paradigms remain at an early stage in this field (Brouwer et al., 2013; Kotton, 2012; Takahashi et al., 2007). Despite ongoing challenges, the key advantage of using autologous iPSCs is the potential to avoid immune rejection (Beers et al., 2017; Kubo, 2012; Nishikawa, Goldstein, \& Nierras, 2008; Takahashi et al., 2007; Yu et al., 2007). In addition, the iPSC-derived cells can perhaps expand to necessary numbers-that is, many billions-to repopulate decellularized human-sized lung scaffolds.

We have previously reported a stepwise differentiation protocol to generate a population of type II epithelial-like progenitor cells from 
human iPSCs (Ghaedi et al., 2013). The type II progenitor cells derived from iPSC were positive for markers (prosurfactant proteins) such as pro-SPC and pro-SPB. In this study, we adapted the differentiation protocol to generate airway progenitor cells from iPSC, based upon recently reported methods with a few modifications (Huang et al., 2014; Longmire et al., 2012; Mou et al., 2012; Wang, Haviland, Burns, Zsigmond, \& Wetsel, 2007). The next goal, was to explore the capacity of iPSC-derived lung progenitor cells to repopulate a decellularized lung scaffold and evaluate cell viability and phenotype of these cells while they were cultured on decellularized lung scaffold.

To determine the feasibility of producing repopulated lungs from iPSC-derived epithelial stem cells, we repopulated decellularized human lung obtained from tissues not suitable for transplantation. The acellular scaffolds were repopulated in the alveolar and airway compartment with iPSC-derived lung progenitor cells, and we assessed the cell attachment, viability, and epithelial phenotype retention on the human scaffolds.

We further generated the engineered rat lung from both endothelial cells and iPSC-derived epithelial cells in a rat bioreactor system (Gilpin, Charest, Ren, Tapias, et al., 2016; Petersen et al., 2010; Petersen, Calle, Colehour, \& Niklason, 2011). The iPSC-lung epithelial cells and rat lung microvascular endothelial cells were introduced to the airway and vascular compartments of acellular rat scaffolds, respectively, and were cultured for 3-5 days in a bioreactor system.

Our study makes further progress in the field of lung tissue engineering, by evaluating the potential suitability of iPSC-derived cells to repopulate clinically applicable matrices. In addition, this technology may provide an ex vivo model for the deeper understanding of human lung biology and lung disease and for studying the interactions between progenitor cells and lung scaffolds.

\section{2 | MATERIALS AND METHODS}

\section{1 | Cultivation of human iPSCs}

The two human episomal iPSC lines (MIRJT7i-mND2-0 and iPS-DF69-9 T.B) utilized in this study were provided by Prof. James A. Thomson, Department of Anatomy, University of Wisconsin-Madison, Madison, WI, USA. Both human iPSC lines were propagated on Matrigel in mTesR medium, with medium changes every day. Undifferentiated iPSCs were passaged onto fresh Matrigel every 4-5 days.

\subsection{In vitro differentiation of iPSCs into alveolar and airway epithelial cells}

The iPSCs were first differentiated toward definitive endoderm (DE) in RPMI 1640 medium supplemented with $100-\mathrm{ng} / \mathrm{ml}$ activin A 2-mM L-glutamine, and 1\% antibiotic-antimycotic for $48 \mathrm{hr}$. Then, 1xB27 supplement and 0.5-mM sodium butyrate were added into the same medium, and cells were cultured in this medium for 3 days (D'Amour et al., 2005; Duan et al., 2010). Subsequently, the DE cells were trypsinized and replated on human extracellular matrix protein (Sigma Aldrich)-coated plates and were differentiated to anterior foregut endoderm (AFE) cells by exposing them sequentially to
dorsomorphin/SB431542 from days 5 to 6, followed by IWP2 / SB431542 from days 6 to 7 (Figure S2) in Iscove's Modified Dulbecco's Medium with 10\% fetal bovine serum, 2-mM L-glutamine, 1-mM nonessential amino acids, 1\% antibiotic-antimycotic (Green et al., 2011; Huang et al., 2014; Mou et al., 2012).

The AFE cells were maintained in lung endoderm differentiation medium consisting of Iscove's Modified Dulbecco's Medium and F12 (3:1) with $10 \%$ fetal bovine serum, 2-mM L-glutamine, 1-mM nonessential amino acids, 1\% antibiotic-antimycotic, 1X B27, 1X N2 supplement, ascorbic acid (50 $\mathrm{\mu g}$; basal medium), supplemented with retinoic acid (0.5 $\mu \mathrm{M})$, bFGF (10 ng/ml), BMP4 (10 ng/ml), CHIR99021 ( $3 \mu \mathrm{M} / \mathrm{ml}$ ), and keratinocyte growth factor (KGF; $10 \mathrm{ng} / \mathrm{ml}$ each) for 7 days (Huang et al., 2014; Longmire et al., 2012).

To differentiate the early lung progenitor cells to the ATII (type II epithelial) progenitor cells, they were cultured in the same basal media describe above supplemented with fibroblast growth factor 10 (FGF10; $10 \mathrm{ng} / \mathrm{ml}$ ), retinoic acid $(0.5 \mu \mathrm{M})$, epidermal growth factor (EGF; $10 \mathrm{ng} / \mathrm{ml}$ ), CHIR99021 (3 $\mu \mathrm{M} / \mathrm{ml})$, and KGF (10 $\mathrm{ng} / \mathrm{ml})$ for another 10 days. At day 25 , the growth factor combination was switched to retinoic acid $(0.5 \mu \mathrm{M})$, EGF $(10 \mathrm{ng} / \mathrm{ml})$, FGF10 (10 ng/ml), and KGF (10 ng/ml) dexamethasone (50 nM), butyryl cGMP (0.1 mM), and isobutylmethylxanthine $(0.1 \mathrm{mM})$ and cells were cultured in this medium, until use (Green et al., 2011; Longmire et al., 2012).

To differentiate the early lung progenitor cells to the proximal airway progenitor cells, at day 15 , the growth factors combination was switched to high concentration of retinoic acid (1 $\mu \mathrm{M})$, RA-supplemented B27, BMP7 (10 ng/ml), KGF (10 ng/ml), EGF (10 ng/ml), dexamethasone (50 nM), butyryl cGMP (0.1 mM), and isobutylmethylxanthine $(0.1 \mathrm{mM}$ ) for 15 days. (Huang et al., 2014; Mou et al., 2012; see detailed protocols in Supporting Information). Following culture protocol for human airway described by Randell's laboratory (Fulcher \& Randell, 2013), on day 32, cells were split with trypsin and reseeded on collagen I/III-coated plates in $\mathrm{BEGM}^{\mathrm{T}}$ medium from Lonza until use (Fulcher \& Randell, 2013).

\section{3 | Primary human airway epithelial cells}

Human airway epithelial cells procured under the University of North Carolina at Chapel Hill Institutional Review Board protocol number 03-1396 were kindly provided by Dr. Scott Randell.

\section{4 | Preparation of decellularized extracellular matrix scaffolds}

All animal experiments were performed with the approval of the Institutional Animal Care and Use Committee of Yale University, and all studies using human lung tissue were approved by the University of Michigan. Sprague Dawley were used as a source for rat donor lungs. Human lungs were obtained from beating-heart donors or warm autopsy as arranged through Gift of Life Michigan. In order to build a "protocol" that could be used for rat, and human lungs with different sizes, we used a decellularization regimen that normalizes to tissue size and operates on a volume of reagent per gram wet weight tissue basis as previously described (Balestrini et al., 2015; Booth et al., 2012). 
Briefly, decellularization was achieved by perfusion with a $0.0035 \%$ Triton $\mathrm{X}-100$ solution followed by sodium deoxycholate solutions of increasing concentration from $0.01 \%$ to $0.1 \%$. The lung was rinsed with sterile phosphate-buffered saline (PBS) and perfused with $30-\mathrm{U} / \mathrm{ml}$ Benzonase Nuclease in 1-M Tris-buffer with 1-mM $\mathrm{MgCl}_{2}$. Lastly, the lobe was rinsed with $0.5 \%$ Triton X-100 solution followed by additional sterile PBS. (See detailed protocols in Supporting Information).

\section{5 | Culture of iPSC-lung epithelial cells onto lung extracellular matrix scaffolds}

a. Human lung scaffold: Donated human lungs that are not suitable for transplantation were used to produce natural lung scaffolds (Booth et al., 2012). The middle-right lobe of a decellularized human lung was mounted in the bioreactor. Five hundred million iPSC-ATII progenitor cells and $500 \times 10^{6}$ iPSC-airway progenitor cells were suspended in $3 \mathrm{ml}$ of epithelial medium and introduced into the scaffold via the airway. Vascular perfusion with epithelial growth media was initiated at $3 \mathrm{ml} / \mathrm{min}$ immediately after cell seeding. The reseeded lung scaffolds were then cultured in the lung bioreactor with no breathing for 4 days in epithelial cell medium described in Figure S7G (see Supporting Information) with the whole volume of culture medium $(500 \mathrm{ml})$ changed every day.

b. Rat lung scaffold: 100 million rat microvascular endothelial cells (RLMEC) were perfused through the vasculature by gravitydriven, pulsatile perfusion, and the lungs were perfused at $1 \mathrm{ml} /$ min for $4 \mathrm{hr}$ until epithelial cell seeding. Each set of EC-seeded lungs were repopulated with 50 million iPS-epithelial cells $\left(25 \times 10^{6}\right.$ iPSC-ATII progenitor cells and $25 \times 10^{6}$ iPSC-airway progenitor cells) suspended in $1.5 \mathrm{ml}$ of via the trachea in the bioreactor and were perfused between 1 and $4.5 \mathrm{ml} / \mathrm{min}$. The engineered rat lungs were maintained in a bioreactor system for 4 days in coculture medium described in Figure S7G, with culture media, changes twice daily (see detailed protocols in Supporting Information).

\section{6 | Real-time quantitative reverse transcription- PCR}

Total RNA was extracted from cells using the RNeasy Mini Kit from Qiagen following the manufacturer's instructions. First-strand complementary DNA (cDNA) was synthesized using SuperScript First-Strand Synthesis System according to manufacturer's protocol (Invitrogen). Reactions were performed in a $25-\mu \mathrm{L}$ volume with $\mathrm{iQ}^{\mathrm{TM}}$ SYBR Green Supermix (Bio-Rad), 1- $\mu \mathrm{L}$ of cDNA, and $200 \mathrm{nM}$ each of forward and reverse primers using iCyler and iQ software. Average of $\mathrm{Ct}$ values from the triplicate PCR reactions for a gene of interest were normalized against the average Ct values for glyceraldehyde-3-phosphate dehydrogenase from the same cDNA sample and expressed relative to normal cadaveric human or rat lung tissue.

\section{7 | Immunofluorescence, immunohistochemistry, and flow cytometry}

Tissues were fixed in formalin for $4 \mathrm{hr}$ and cells were fixed in $4 \%$ paraformaldehyde for $\mathbf{2 0} \mathrm{min}$. For immunofluorescence, fixed cells or tissue sections after deparaffinization were permeabilized with $0.1 \%$ Triton $\mathrm{X}-100$ in PBS for $15 \mathrm{~min}$, blocked in 3\% BSA in PBS for $60 \mathrm{~min}$ at $\mathrm{RT}$, and then incubated in primary antibody overnight at $4{ }^{\circ} \mathrm{C}$ and secondary antibody for $2 \mathrm{hr}$ at RT. After washing with PBS, the cells were incubated with DAPI $(1 \mu \mathrm{l} / \mathrm{ml})$ for $1 \mathrm{~min}$ and mounted with PVA-DABCO cover slipping solution and slides.

For flow cytometry, $5 \times 10^{5}$ cells were resuspended in $250 \mu \mathrm{l}$ of fixation/permeabilization solution from a BD Bioscience cytofix/ cytoperm kit for $20 \mathrm{~min}$. After blocking for $30 \mathrm{~min}$ on ice, the cells were incubated with corresponding primary antibody in the blocking solution for $30 \mathrm{~min}$ on ice. The cells were resuspended in $350 \mu \mathrm{l}$ of Perm/Wash buffer after incubation with the corresponding conjugated secondary for $30 \mathrm{~min}$ on ice, washed twice, and analysed by flow cytometry.

\subsection{Statistical analyses}

All statistical analyses were done with the software Origin (OriginLab, Northampton, MA). The data were expressed as mean \pm standard error of measurement. The $t$ tests were performed to evaluate whether two groups were significantly different from each other. The $p$ values less than .05 (two-tailed) were considered statistically significant.

Complete methods can be found in the Supporting Information.

\section{3 | RESULTS}

\subsection{Generation of human lung epithelial cells from iPSCs}

We previously reported a stepwise differentiation method to generate $\mathrm{DE}, \mathrm{AFE}$, and subsequently, early lung progenitor cells from human iPSCs (Ghaedi et al., 2013). To improve lung epithelial cell phenotype, in this work, we modified previously published protocols (Green et al., 2011; Longmire et al., 2012; Mou et al., 2012; Wong et al., 2012) and generated a protocol to derive both alveolar and airway progenitor cells from iPSCs, by following the timing and coordination of the signalling pathways in lung development (Figure 1a).

As in previously published studies (Green et al., 2011; Kubo et al., 2004), $>85 \%$ endodermal cells were generated from human iPSCs by exposing them to saturating concentrations of activin A during the first 5 days of differentiation (Figures 1c,h and S1). In the second step, we differentiated the DE to AFE by exposing them sequentially between days 5 and 7 to combinations of the small molecule inhibitors. (Figures 1a,d,i and S2A-D; Huang et al., 2014). To specify lung cell fate, at day 8 , the medium was switched to lung endoderm differentiation medium containing bFGF, BMP4, CHIR, and KGF for 7 days (Figures S2A and S3). When we examined the expression of the early lung marker NKX2.1 at day 15 of differentiation, immunostaining and quantitative PCR results showed that up to $30 \%-40 \%$ of cells were positive for this marker (Figures 1e,j and S2A-C). 


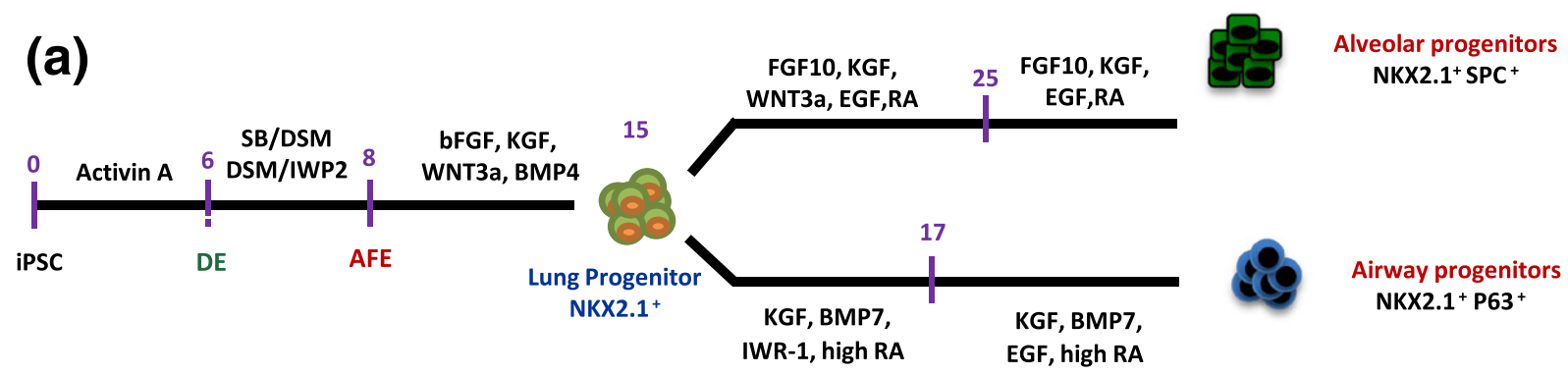

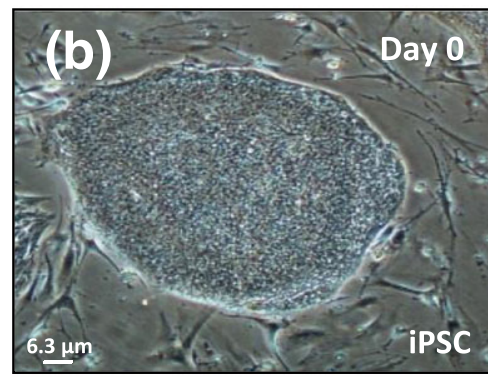
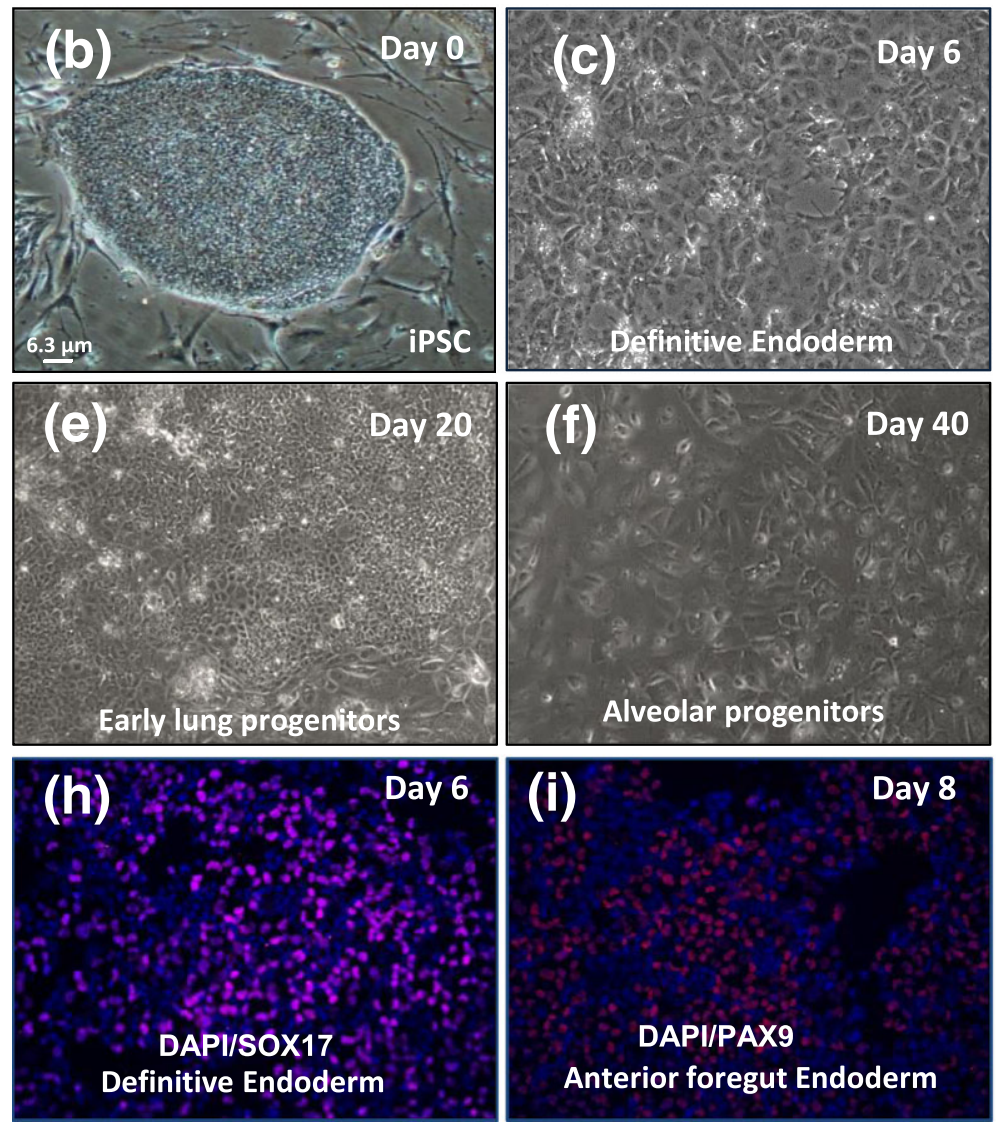
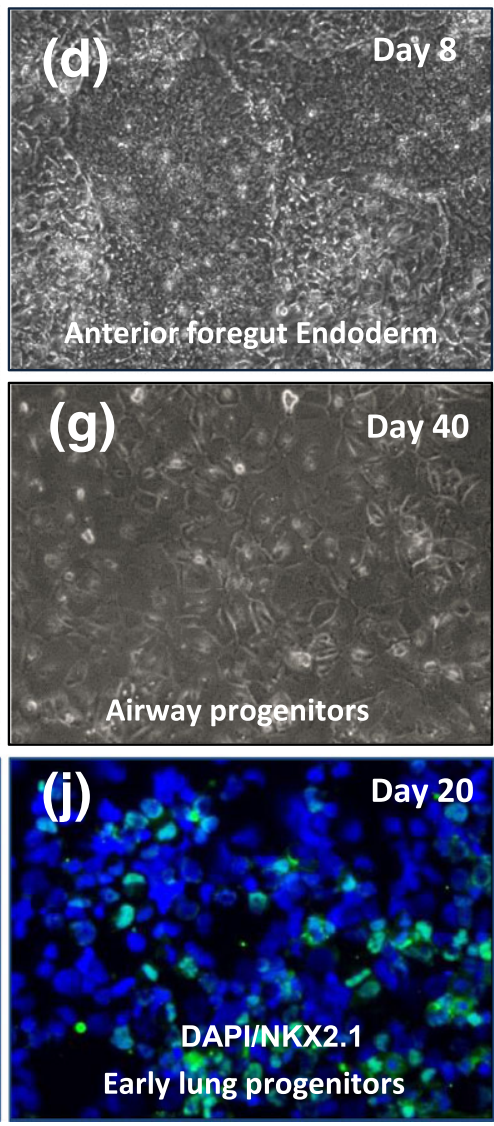

FIGURE 1 Generation of lung epithelial cells from human induced pluripotent stem cell (iPSC) in vitro. (a) Schematic for differentiation protocol of human iPSC to alveolar and airway progenitor cells in vitro. (b) Phase-contrast images of human iPSC, (c) definitive endoderm (DE), (d) anterior foregut endoderm (AFE), (e) early lung progenitor cells at day 20, ( $\mathrm{f}-\mathrm{g}$ ) alveolar and airway progenitor cells at day 40 (scale bar $=6.3 \mu \mathrm{m}$ applies to panels b-g). (h-j) immunofluorescent images of differentiated cells from iPSC for (h) SOX17 (endoderm marker) at day 6, (i) PAX9 (anterior foregut endoderm marker) at day 8 and NKX2.1, early marker of lung progenitor cells at day 20 (scale bar $=31 \mu \mathrm{m}$ applies to panels $\mathrm{h}$-i and $21 \mu \mathrm{m}$ to panel j). DAPI = 4',6-diamidino-2-phenylindole; EGF = epidermal growth factor; KGF = keratinocyte growth factor; FGF10 = fibroblast growth factor 10

To differentiate early lung progenitor cells into type II progenitorlike cells, we cultured the progenitor cells at day 15 in differentiation media containing KGF, FGF10, RA, CHIR, EGF for another 2 weeks (Huang et al., 2014), after which CHIR was removed from the differentiation cocktail for the rest of differentiation (Figure S3). At day 40 of differentiation, the cells, now termed "ATII progenitor cells," shown to express type II cell markers (Longmire et al., 2012; Figures $1 \mathrm{f}$ and S7). Immunofluorescence staining and quantitative reverse transcription-PCR (qRT-PCR) showed the iPSC-ATII progenitor cells were positive for type II markers, including surfactant protein C (SPC) and NKX2.1, and that a small fraction of the cells expressed type I surface markers, T1a (Figure 2A-E).
To enhance the differentiation of lung progenitors into a phenotype more consistent with airway progenitor cells (i.e., NKX2.1 $1^{+} / \mathrm{P} 63^{+}$ or $\mathrm{NKX} 2.1^{+} / \mathrm{CK} 5^{+}$) rather than ATII progenitor cells, at day 15 , the growth factor combination was switched to BMP7, KGF, high-concentration retinoic acid, and WNT antagonist (IWR-1). After 17 days, the medium was switched to BEGM from Lonza (Mou et al., 2012; Figures S2A and S3). Immunostaining at day 40 showed cells now termed "airway progenitor cells" (Figure 1g), stained positive for several airway progenitor cell markers such as NKX2.1, P63, CK5, and CK14 (Figure 2f-i). Furthermore, quantitative RT-PCR revealed that $\mathrm{CK} 5$ and P63 were expressed in iPSC-airway progenitor cells (up to $50 \%$ of the cells). However, these cells expressed lower levels of lung 


\section{Alveolar Progenitor cells}
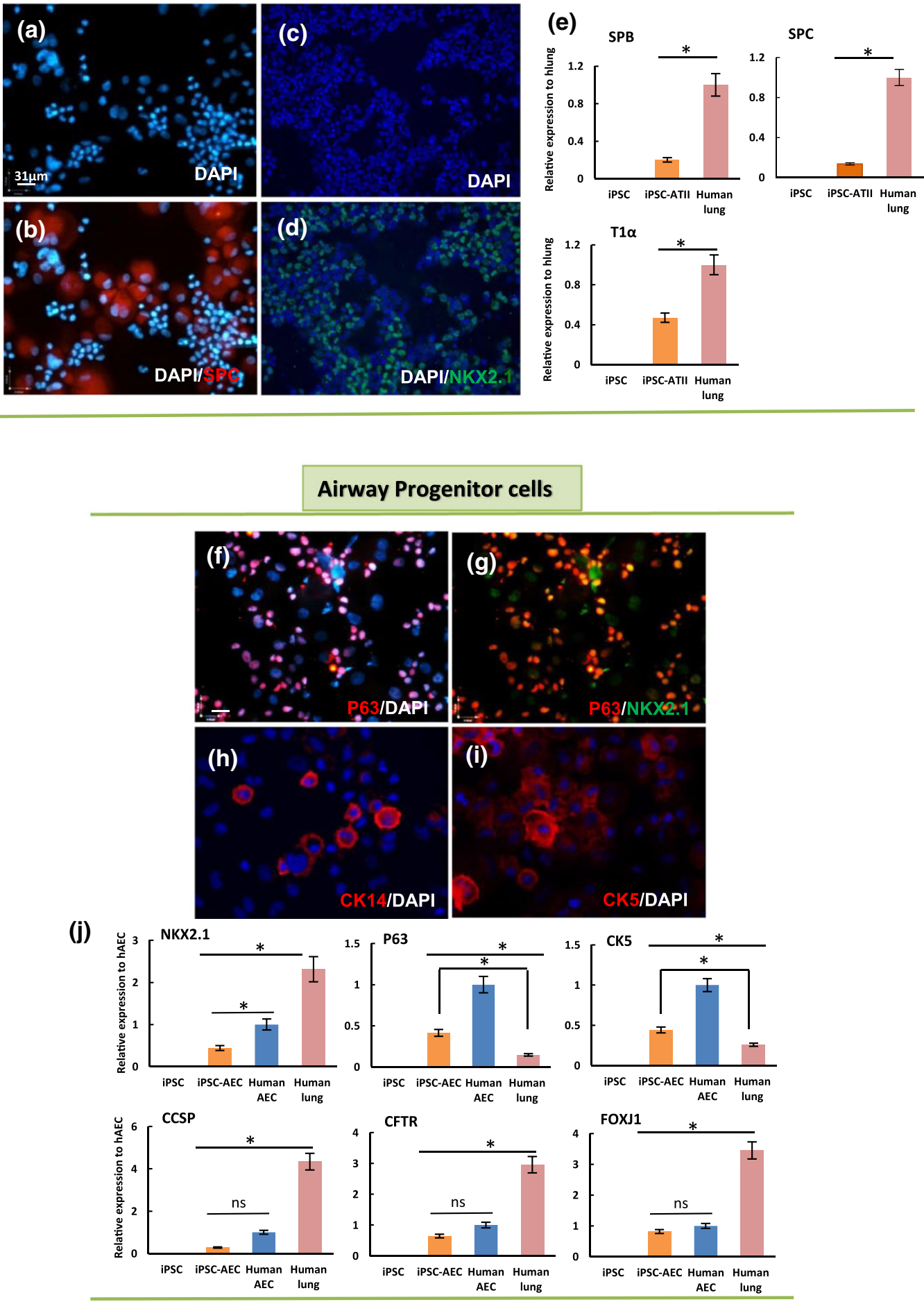

FIGURE 2 Generation of lung alveolar and airway epithelial progenitor cells from human induced pluripotent stem cell (iPSC) in vitro. (a-d) immunofluorescent images of iPSC-alveolar progenitor cells at day 40 (cytocentrifuged prepared), illustrating positive staining for (a) 4',6diamidino-2-phenylindole (DAPI), (b) DAPI-SPC, (c) DAPI, and (d) DAPI-NKX2.1. (e) Quantitative reverse transcription-PCR (qRT-PCR) analysis of type II epithelial cell markers in iPSC-lung progenitor cells at day 40; surfactant proteins B and C, and T1a (a type I cell marker). (f-i) immunofluorescent staining for airway markers in iPSC-airway progenitor cells (cytocentrifuged prepared) at day 40: (f) DAPI-P63, (g) NKX2.1 and P63, (h) DAPI-CK14, (i) DAPI-CK5 (scale bar $=31 \mu \mathrm{m}$ applies to panels a-d and f-i). (j) qRT-PCR analysis of airway epithelial cell markers in iPSC-lung progenitor cells at day 40. For qRT-PCR, values from three independent experiments from the triplicate PCR reaction for each gene were normalized to average glyceraldehyde-3-phosphate dehydrogenase $\mathrm{Ct}$ values from the same cDNA samples. The relative expression of each gene was then calculated to normal human lung (hlung) and airway cells isolated from human trachea (hAEC; $n=3$ independent replicates for qRT-PCR, error bars indicate \pm SEM and *indicates $p<.05$ and ns indicates no significant difference). CCSP = Clara cell secretory protein; CFTR $=$ cystic fibrosis transmembrane conductance regulator; hAEC = human airway epithelial cells 
epithelial markers as compared with freshly isolated human airway cells and human lung RNA (Figure $1 \mathrm{j}$ ).

Together, these results indicated differentiation of human iPSC cells into lung epithelial progenitor cells expressing main alveolar and airway markers such as SPC, NKX2.1, P63, and CK5. Given these observations, we elected to determine the fate of these cells when seeded into acellular lung scaffolds (Gomperts \& Strieter, 2007; Rackley \& Stripp, 2012; Rock et al., 2011).

\subsection{Repopulating human lung tissue scaffolds with iPSC-lung epithelial cells}

Next, we explored the capacity of iPSC-derived lung progenitor cells to repopulate the airway compartment of a decellularized lung ECM. Acellular middle right lobes of the human lung were reseeded with an iPSC-ATII progenitor cells and iPSC-airway progenitor cells. In repopulated constructs, cell coverage was assessed by histological staining of multiple sections. haematoxylin and eosin staining showed that iPSC-derived epithelial cells were able to diffusely repopulate alveolar structures and conducting airways within the intact lung scaffold (Figure $3 b-c)$. To engineer a functional lung repopulating a native lung matrix with sufficient cell numbers in appropriate anatomic locations is required (Gilpin, Charest, Ren, \& Ott, 2016; Gilpin, Charest, Ren, Tapias, et al., 2016). Immunostaining showed some cells in the alveolar compartments of the repopulated lung expressed the type II cell markers, SPC, and NKX2.1, and cellular expression of these markers was robust at day 4 of culture (Figure $3 f-k$ ). We also observed that cells located in the airway compartments were positive for airway epithelial markers such as Clara cell secretory protein (CCSP) and Forkhead box protein J1 (FOXJ1), confirming the commitment of the iPSC-airway progenitor cells to airway phenotype after scaffold culture (Figure 3l-q). However, the cell attachments were not site specific and in some sections of the repopulated lung, cells which landed in alveolar compartment expressed airway markers or cells that landed in airway compartments expressed alveolar cells markers. The iPSC-derived epithelial cells used for repopulating the acellular lung scaffold are still progenitor cells at the time of seeding. Long-term culture, in bioreactor system, might help these cells to differentiate into the right cell type based on the signals that they receive from the scaffold or growth media. Immunostaining for proliferating cell nuclear antigen and caspase-3 (apoptotic marker) showed that the cells were able to replicate in the acellular scaffold and displayed few markers of apoptotic cell death during the culture period. This confirmed that our bioreactor culture system successfully maintained adequate nutrient transfer for the ex vivo repopulated lungs and facilitated the continued growth and expansion of the iPSC-derived progenitor cells (Figure 4a,b)

To quantify the expression of cell markers the repopulated human lung tissues were assayed by qRT-PCR for expression levels of various epithelial markers and compared with mRNA derived from native human lung. We identified a trend for higher expression of lung epithelial markers such as SPC, T1a, CK5, and Mucin 5 AC in iPSC-derived lung progenitor cells cultured in the lung scaffold, compared with the same cells grown on traditional culture plates. The expression of FOXJ1 and P63 was significantly higher in cells cultured in the lung scaffold compared with the same cells cultured on tissue culture plate.
These data suggest that the 3D acellular lung and the physiological relevant culture system improved the differentiation and maturation of the iPSC-derived cells (Figure 4c). However, in most cases, levels of epithelial gene expression in the engineered lung were significantly lower than those in native lung samples.

\subsection{Engineered rat lung from human iPSC-lung epithelial cells and rat microvascular endothelial cells}

To generate the lung tissue from both epithelial and endothelial cells, RLMEC were delivered into the vascular compartment of the decellularized rat lung. Subsequently, ATII and airway progenitor cells from human iPSC were introduced via the airways to repopulate the alveolar and airway compartment of the scaffold.

When the cell coverage and proliferative capacity of the cells within the matrix were assessed, haematoxylin and eosin staining showed that RLMEC and iPSC-derived cells were able to repopulate alveolar-airway lung structures and vascular compartment within the scaffold (Figure 5a,b). Immunofluorescence showed epithelial cells present in the alveolar compartments expressed alveolar type II markers, SPC, SPB, and NKX2.1 (Figure 5c-e) while cells within the airway compartments expressed airway markers, CCSP, FOXJ1, and P63 (Figure 5f-h). As we observed in repopulated human lung with iPSCepithelial progenitor cells, the cell attachments were not site specific. The majority of cells within the matrix expressed proliferating cell nuclear antigen, while few of them displayed caspase-3, indicating cells were able to proliferate in the acellular rat matrix (Figure $5 \mathrm{i}, \mathrm{j}$ ). The qRTPCR revealed the pronounced expression of different alveolar (SPC and NKX2.1) and airway epithelial (P63, CK5, CCSP, and Mucin5AC) markers in the engineered rat lung compared with the same cells grown on tissue culture plate, indicating the natural scaffold and coculture with endothelial cells may have improved the expression of epithelial cell markers (Figure $5 \mathrm{~m}$ ). However, the differences in gene expression level were not significant for most of epithelial markers, except for the P63 gene.

In the vascular compartment, rat microvascular endothelial cells repopulated the vascular matrix and expressed several functional markers. Immunofluorescence staining for markers CD31 and endothelial nitric oxide synthase (eNOS) showed extensive expression of these markers in repopulated rat lungs (that also contained epithelial cells; Figure 5k,l). By qPCR, engineered lungs containing RLMEC also expressed pronounced levels of eNOS, CD31, and CD144 in engineered rat lung. We observed a high level of eNOS in engineered rat lung, which is important for endothelial cell function and inhibition of intravascular coagulation (Figure $5 \mathrm{~m}$ ).

\subsection{Assessment of cellular response to different acellular lung scaffolds}

As part of our post-production assessment, we compared the expression levels of several lung epithelial markers in iPSC-derived progenitor cells when seeded into decellularized human and rat lung scaffolds. Comparison of the engineered rat lung with engineered human lung for attachment of cells showed that the iPSC-derived cells adhered to the rat lung scaffold in a manner 

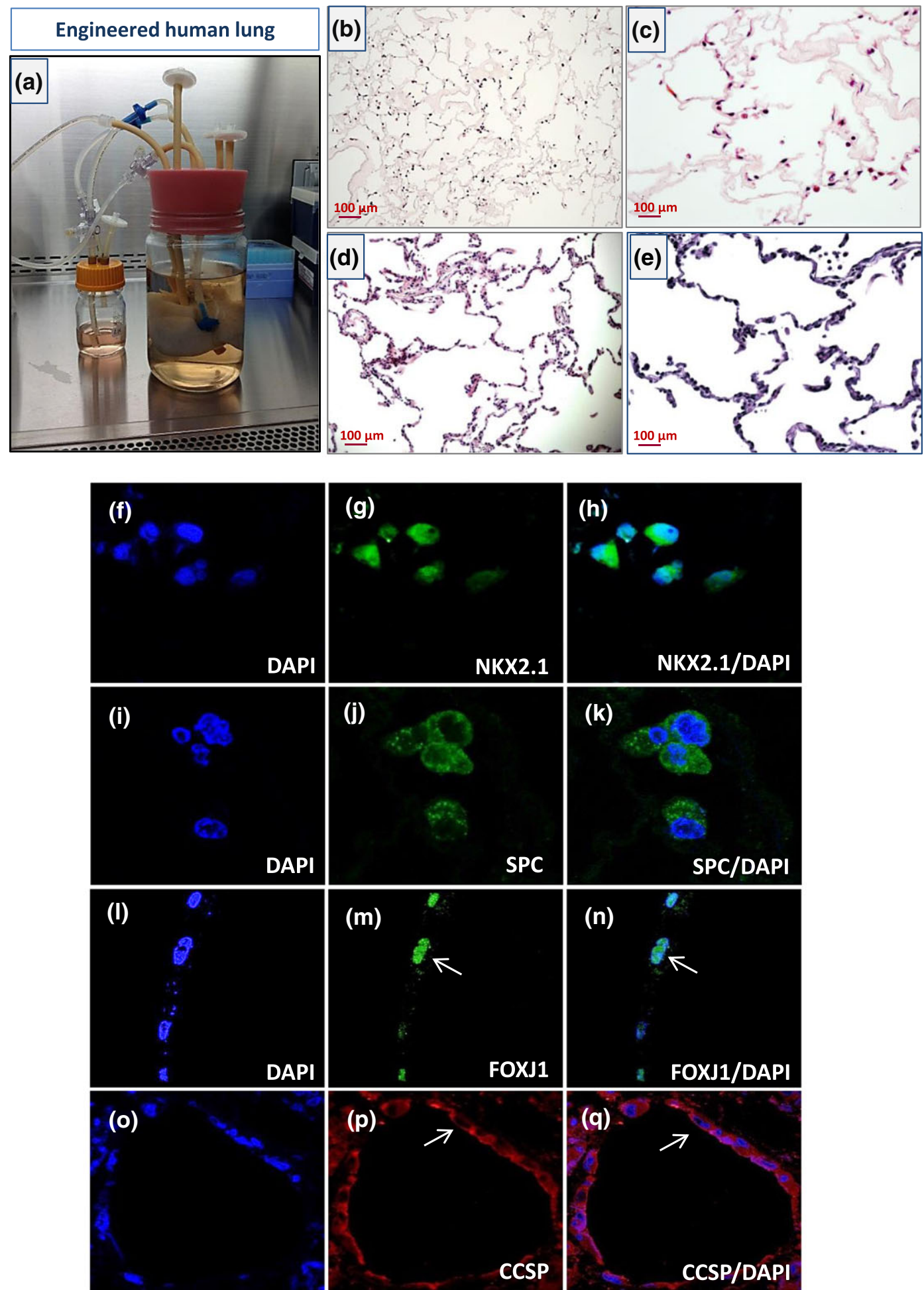

FIGURE 3 Functional culture of engineered human lung made of human lung scaffold and iPSC-derived lung epithelial progenitor cells in a bioreactor system. (a) Recellularized human lung scaffold with iPSC-lung epithelial progenitor cells in a bioreactor culture system. (b-c)

Representative images of haematoxylin and eosin sections of recellularized human lung after 4 days of culture. (d-e) Haematoxylin and eosin images of a native human lung (scale bar $=100 \mu \mathrm{m}$ applies to panels $\mathrm{b}-\mathrm{e}$ ). ( $\mathrm{f}-\mathrm{q}$ ) Immunofluorescent images of recellularized lung tissue at day 4 for (f-h) 4 ',6diamidino-2-phenylindole (DAPI) and NKX2.1, (i-k) DAPI and surfactant protein C (SPC), (I-n) DAPI and Forkhead box protein J1 (FOXJ1), and (o-q) DAPI and Clara cell secretory protein (CCSP; scale bar $=63 \mu \mathrm{m}$ applies to all panels $\mathrm{f}$-q; arrows show the cells landed in airway express FOXJ1 and CCSP)

similar to human lung tissue. Averaged data for expression of several lung markers indicated a similar level of gene expression for P63, CCSP, and Mucin 5 AC in iPSC-derived lung progenitor cells that were cultured on rat or human lung scaffolds (Figure 6a-h).
Indeed, there were no significant differences in expression levels of lung markers in iPSC-derived lung progenitor cells cultured on the human matrix as compared with cells cultured in rat lung scaffolds (Figure 6a-h; $p<.05$ ). 

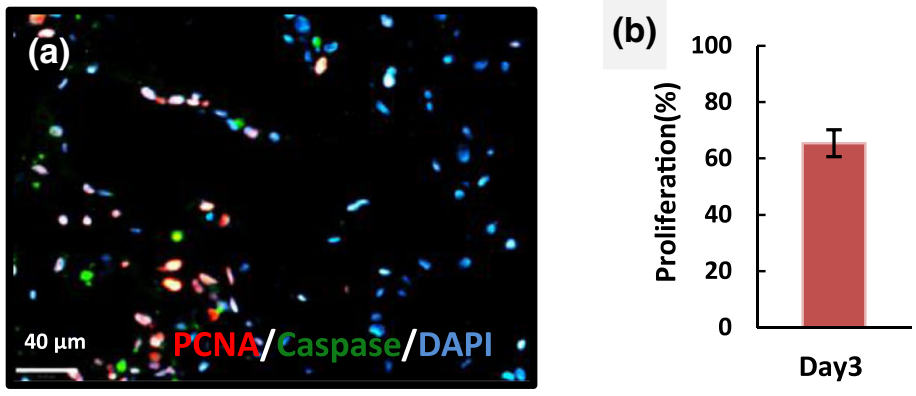

(c)
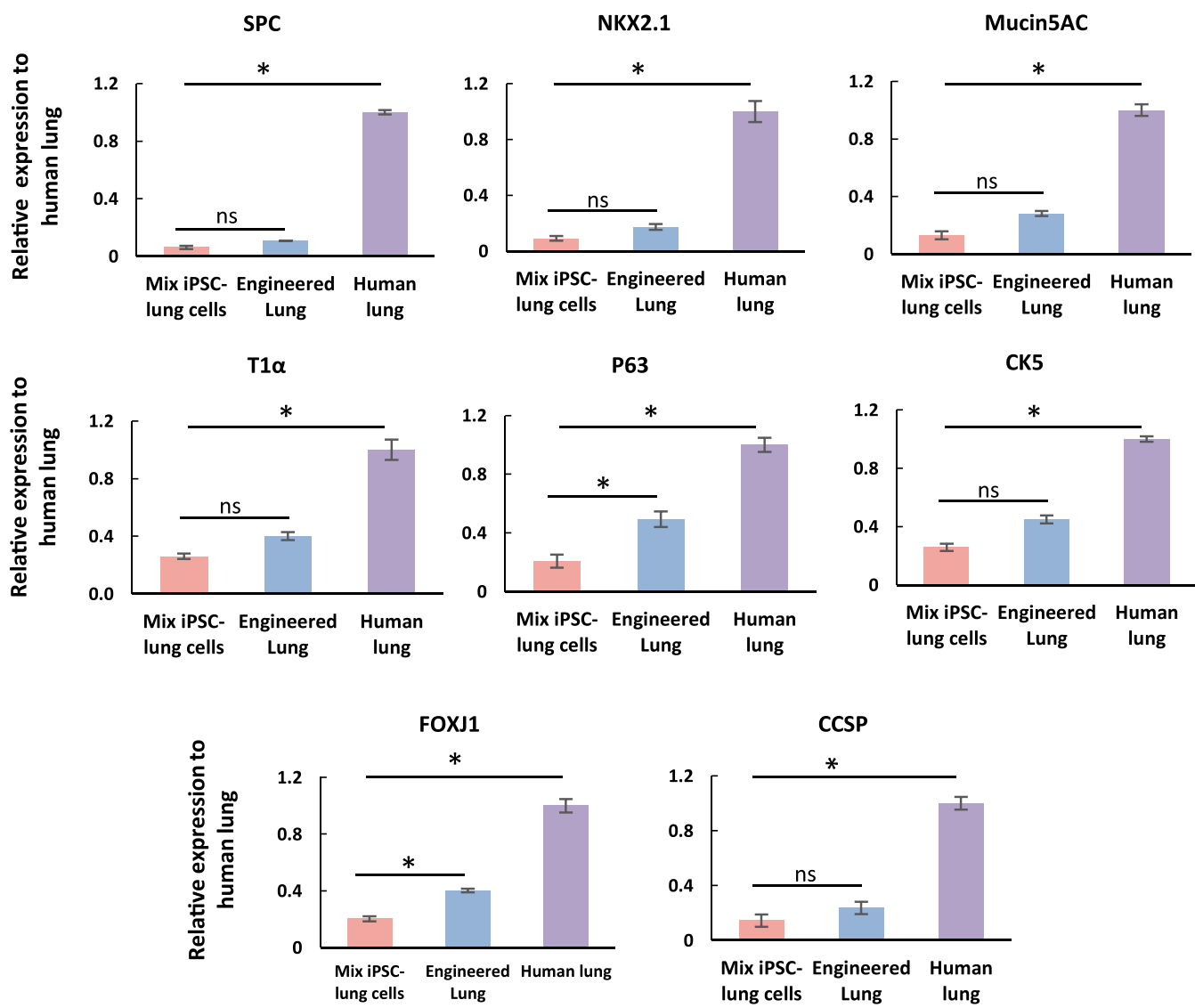

FIGURE 4 Proliferation and gene expression of induced pluripotent stem cell (iPSC)-derived progenitor cells in repopulated human lung matrix. (a) Immunostaining for proliferating cell nuclear antigen (PCNA; red), caspase (green), and 4',6-diamidino-2-phenylindole (DAPI) in an engineered human lung at day 4 (scale bar $=40 \mu \mathrm{m}$ ) and (b) quantification of percentage of PCNA+ nuclei ( $y$ axis, percentage proliferation based on the number of positive nuclei stained for PCNA compared with total cell numbers in three high power fields). (c) Quantitative reverse transcription-PCR analysis of epithelial markers in recellularized lung tissue with iPSC-lung progenitor cells following 4 days of culture. The expression of lung alveolar and airway epithelial cell markers are normalized to glyceraldehyde-3-phosphate dehydrogenase levels, and relative expression of each gene was calculated to normal human lung tissue. ( $n=3$ tissue samples analysed/lung for quantitative reverse transcription-PCR and error bars indicate $\pm \mathrm{SEM},{ }^{*}$ indicates $p<.05$ and ns indicates no significant difference). CCSP = Clara cell secretory protein; FOXJ1 = Forkhead box protein J1; $\mathrm{PCNA}=$ proliferating cell nuclear antigen; $\mathrm{SPC}=$ surfactant protein $\mathrm{C}$

\section{4 | DISCUSSION}

A key challenge in producing human-sized engineered lung tissues is to reliably generate large quantities of epithelial and endothelial cells for the redistribution to their original locations in the organ (Petersen, Calle, \& Niklason, 2011; Rackley \& Stripp, 2012). In effort to address this limitation, we developed a method for differentiation of iPSCs to alveolar-like and conducting airway epithelial-like cells, at scale
(Ghaedi et al., 2013; Huang et al., 2013; Ott \& Mathisen, 2011; Weiss et al., 2013).

The embryonic respiratory system originates from the $D E$ (Mou et al., 2012; Rackley \& Stripp, 2012) and differentiates into many kinds of specialized lung epithelial cells. Using our previous protocol, we were able to generate more than $85 \%$ endodermal cells from human iPSCs by exposing them to saturating concentrations of activin A during the first 5 days of differentiation. Later, during development, 

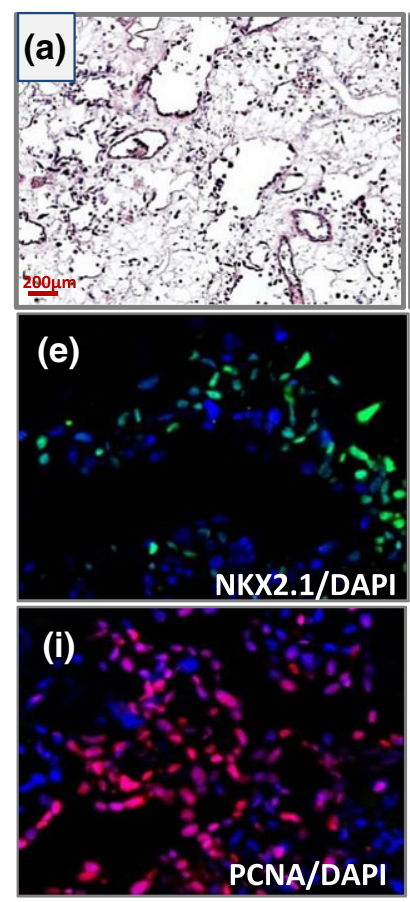
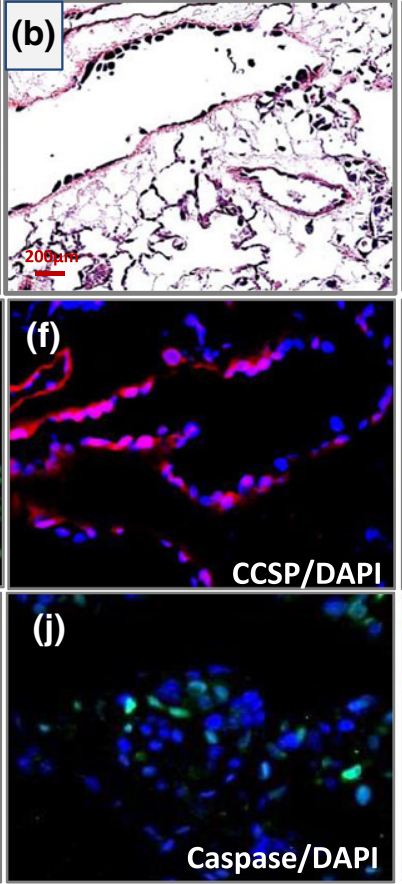
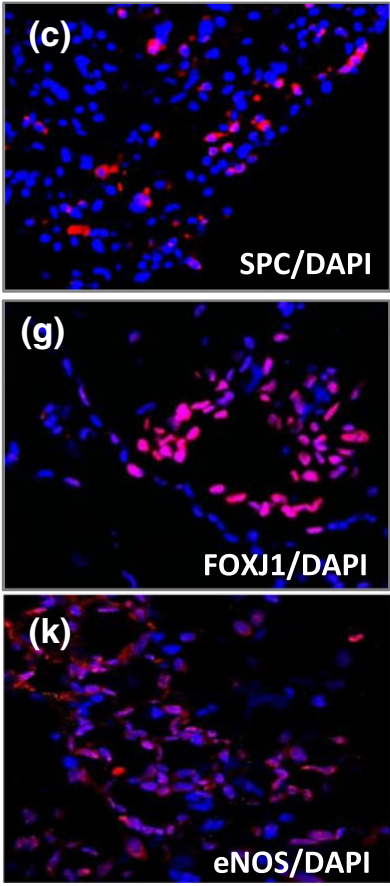
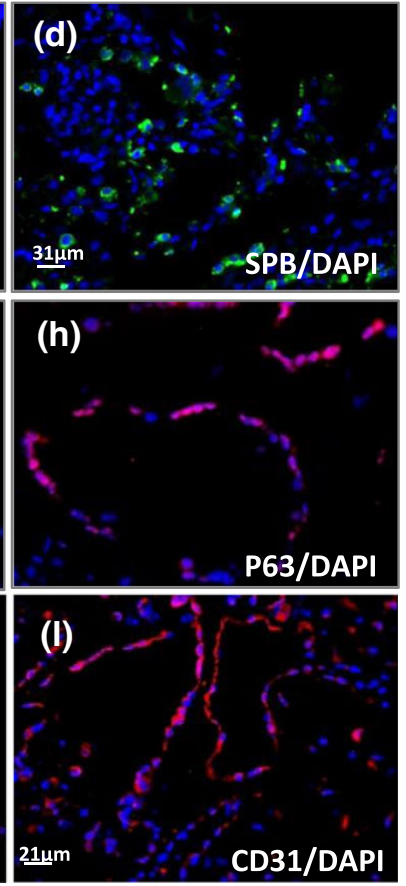
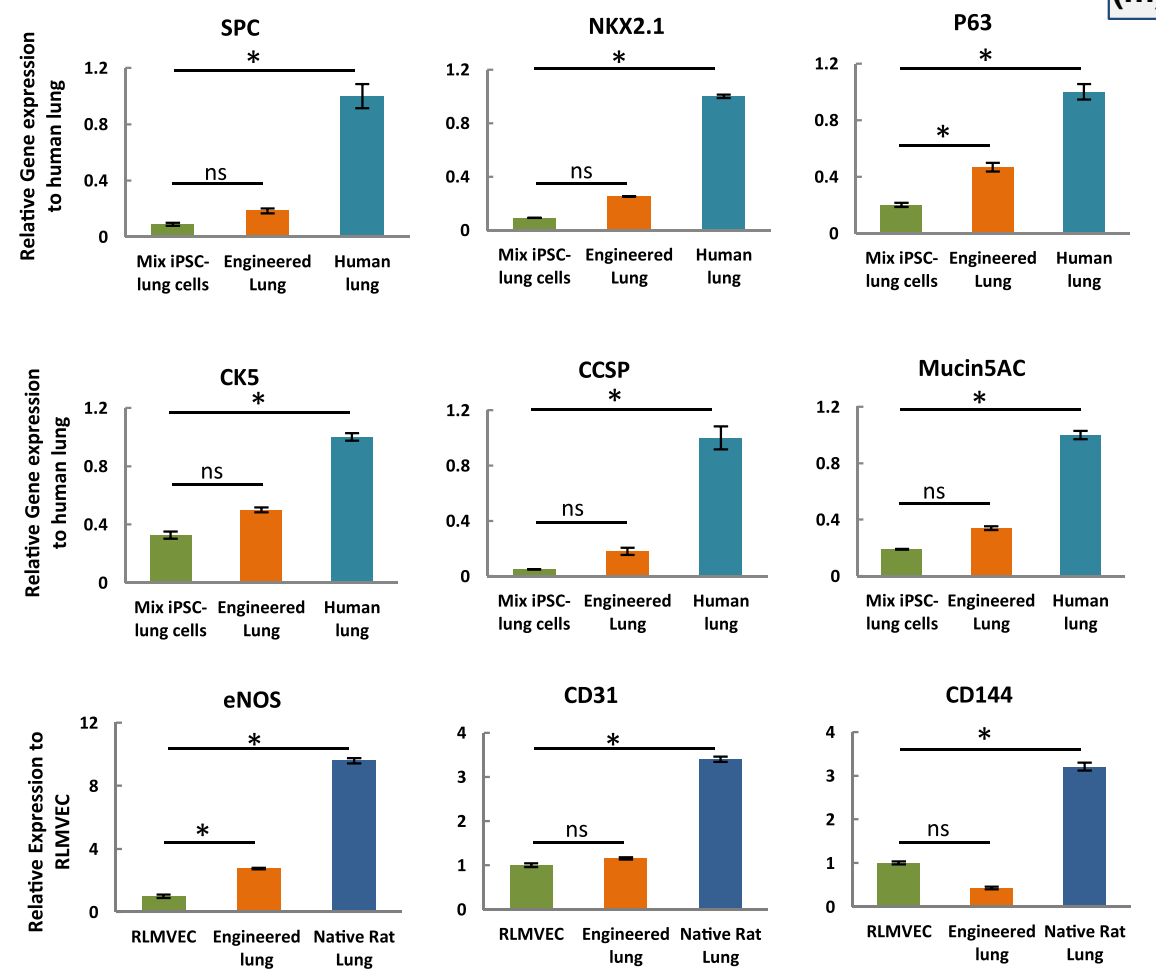

FIGURE 5 Characterization of engineered rat lung from human iPSC-lung epithelial progenitor cells cultured in the rat bioreactor system. (a-b) Representative H\&E staining of engineered rat lung after 4 days of culture (scale bar $=200 \mu \mathrm{m}$ for a-b panels). (c-l) Immunofluorescence analysis of human lung epithelial cell markers and rat endothelial cell markers in an engineered rat lung at day 4 of culture. (c) surfactant protein $C$ (SPC), (d) surfactant protein B (SPB), (e) NKX2.1, (f) Clara cell secretory protein (CCSP), (g) Forkhead box protein J1 (FOXJ1), (h) P63, (i) proliferating cell nuclear antigen (PCNA), (j) caspase (scale bar $=31 \mu \mathrm{m}$ applies to all panels except panel I), (k) endothelial nitric oxide synthase (eNOS), and (I) CD31 (scale bar $=21 \mu \mathrm{m})$. $(\mathrm{m})$ Quantitative reverse transcription-PCR analysis of human lung alveolar (NKX2.1 and SPC) and airway (P63, CK5, CCSP, and Mucin 5 AC) epithelial markers and of rat endothelial cell markers (eNOS, CD31, and CD144) at day 4 of culture. The Ct values of each gene were normalized to glyceraldehyde-3-phosphate dehydrogenase, and the relative expression of each gene was calculated to the human or rat lung tissue control. (All error bars represent \pm SEM. *indicates $p<.05$ and ns indicates no significant difference) 
(a)

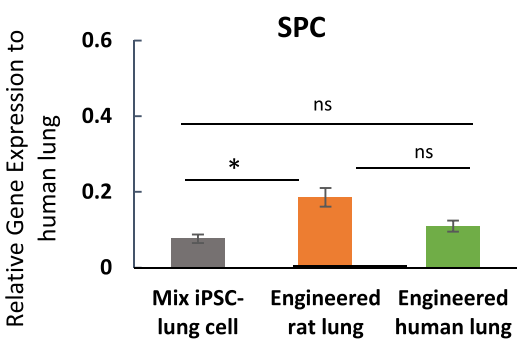

(c)

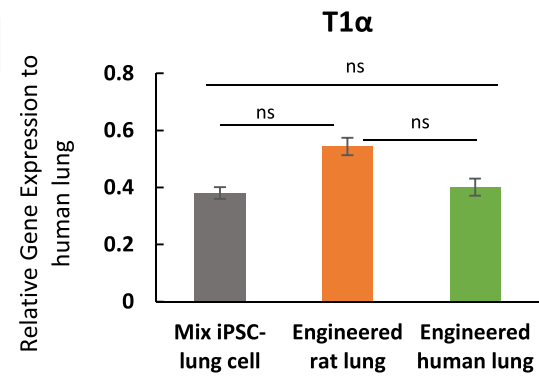

(e)

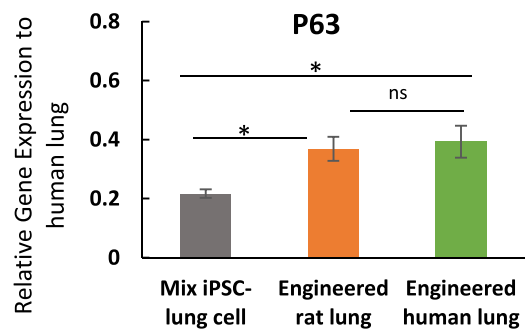

(g)

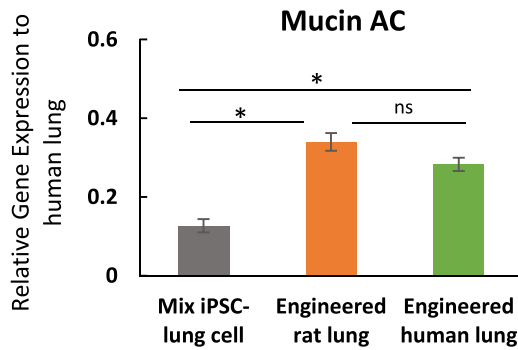

(b)

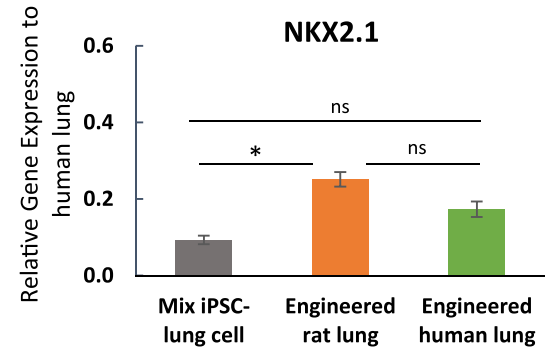

(d)

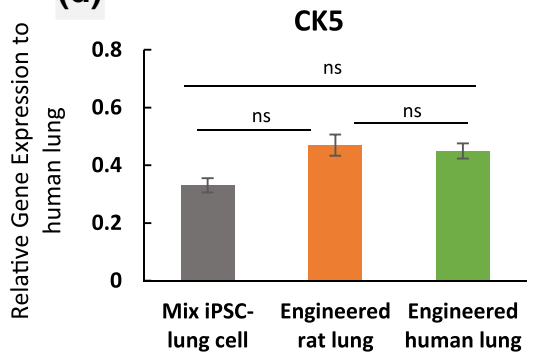

(f)

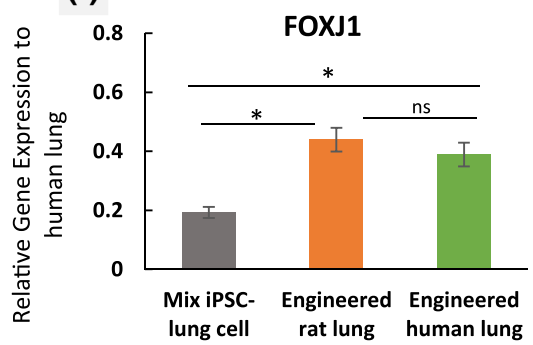

(h)

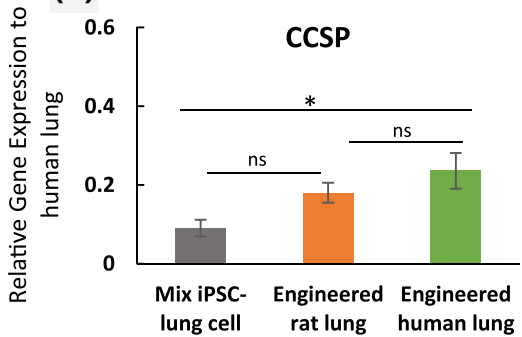

FIGURE 6 Assessment of expression of lung epithelial markers in rat and human lung scaffolds. (a-c) Quantitative reverse transcription-PCR analysis of human lung alveolar epithelial markers (a) surfactant protein C (SPC), (b) NKX2.1, (c) T1a, and (d-h) human lung airway epithelial markers (d) CK5, (e)P63, (f) Forkhead box protein J1 (FOXJ1), (g) Mucin 5 AC, and (h) Clara cell secretory protein (CCSP) in iPSC-derived lung progenitor cells, cultured into the decellularized rat and human lung scaffold compared with native human lung. The expression of each lung epithelial marker was normalized to glyceraldehyde-3-phosphate dehydrogenase levels and relative gene expression to normal human lung tissue were calculated for each gene. ( $n=3$ of iPSC-lung cell samples before seeding, engineered rat and human lung samples were analysed. Error bars represent the \pm SEM, ${ }^{*}$ indicates $p<.05$ and ns indicates no significant difference)

the $\mathrm{DE}$ folds to form the gut tube and is patterned along the anteriorposterior and dorsal-ventral axes (Zorn \& Wells, 2009). Paracrine signals from the surrounding tissues result in specification of the lung field in the ventral AFE, which can be first identified by expression of the transcription factor NKX2.1 (Green et al., 2011; Longmire et al., 2012). Therefore, in the second step, we sought to differentiate the DE further to AFE by dual inhibition of TGF- $\beta / B M P$ and Wnt signalling in iPSC-derived DE (Green et al., 2011; Longmire et al., 2012). Then, to specify lung cell fate and generate NKX2.1 cells, we exposed cells to the combination of bFGF, BMP4, CHIR (Wnt signalling), and KGF.
The choice of growth factor combination was driven from previous findings (Ghaedi et al., 2013; Huang et al., 2014; Longmire et al., 2012; Mou et al., 2012), which showed that the FGF and BMP4 family members KGF and WNT3a are implicated in providing signals during embryogenesis for patterning into lung endoderm.

The human lung features major differences in the composition of the epithelium along its proximodistal axis. The luminal epithelium of trachea, bronchi, and bronchioles is largely composed of ciliated, basal, and Clara-like (or Club) cells. The most distal region of the lung is organized into a complex system of alveoli that are comprised of type 
II and type I epithelial cells (Ali et al., 2002; Garcia et al., 2012; Hashimoto et al., 2012; Rawlins \& Hogan, 2006). Early lung endoderm cells (NKX2.1+) are multipotent, and their fate is dependent upon signals that control proximal versus distal cell fates.

To differentiate the early lung progenitor cells into the type II progenitor cells, we cultured the progenitor cells in differentiation media containing KGF, FGF10, RA, CHIR, and EGF for another 15 days after which $\mathrm{CHIR}$ was removed from the differentiation cocktail for the rest of differentiation. To enhance the differentiation of NKX2.1 $1^{+}$progenitors into airway progenitor cells, the growth factor combination in induction medium was switched to BMP7, KGF, a high concentration of retinoic acid. We found low WNT signalling pathway along with high RA and BMP7 augmented the airway progenitor cells. Together, our data show that using our modified conditions for human type II and airway differentiation, we successfully converted the AFE cells into type II and airway epithelial progenitor cells with good efficiency. Quantitative RT-PCR also confirmed that these iPSC-derived epithelial-like cells were capable of proliferating for several passages (up to P4), and we generated tens of millions of cells with which to seed acellular human matrix scaffolds in this report. However, the differentiated cells at this stage were still multipotent stem cells, and therefore, these cells were the ideal cells for reseeding the airway structures in decellularized human lung scaffolds.

In the acellular human scaffolds, the iPSC-derived progenitor cells repopulated the scaffold in both airways and the alveolar compartment, forming a repopulated tissue that resembled, to some degree, human lung ex vivo, in a bioreactor system. In this study, we were also able to demonstrate the feasibility of producing an engineered rat lung containing human iPSC-derived epithelial progenitors and rat lung microvascular endothelial cells. In the rat scaffold, progenitor cells derived from iPSC were distributed from airway to alveoli, and these engineered lungs displayed much of the microarchitecture of native rat lung.

In the previous report (Gilpin et al), rat lung matrices were repopulated with early lung progenitor cells that were specified to anterior foregut but which displayed very few markers of differentiated lung epithelium (Gilpin et al., 2014). In this current report, we repopulate both human and rat lung tissues using iPSC-derived epithelial progenitors. In contrast to the previous report, in this study, we engineered human lung tissues using two types of iPSC-derived epithelial progenitor cells, including type II-like progenitor cells and airway basal-like cells.

Although these results are encouraging, multiple issues remain to be addressed. For example, to reliably generate large quantities of well-differentiated epithelial cells from iPSCs, the differentiation protocol must be further improved. In future studies, we will test the addition of other combinations of growth factors on the improvement of the differentiation and maturation of iPSC-derived cells. Differentiation of iPSC cells on soft substrates as well as applying mechanical cell stretching during directed differentiation may improve cell growth and epithelial phenotype. In addition, cell differentiation and ciliation may be improved in air-liquid interface during whole-lung culture.

Moreover, the use of stem or progenitor cells for recellularization requires long-term in vitro culturing for cellular proliferation, maturation, and attachment, which must be standardized in the future and also be made affordable. Moving forward, one of the next challenges in lung tissue engineering is the creation of an engineered lung tissue that is functional after implantation in vivo. For successful long-term gas exchange, a formation of good barrier function in the engineered lung is required. The integrity of the physical barrier function in the lung is provided by epithelial and endothelial cells that are mature and express the adhesive molecules to form tight intercellular junctions. Improved strategies for generating more mature vascular endothelium and epithelium, so as to better maintain barrier function and decrease propensity for bleeding and vascular leak, are necessary. Sufficient secretion of surfactants is also required for lung function, to prevent alveolar collapse and preserve bronchiolar patency. Production of surfactant by fully differentiated type II alveolar cells would enable lung inflation without exogenous surfactant.

Considering the potential, distant future clinical application of engineered lungs, other critical issues such as anticoagulation, endothelialization with patient specific endothelial cells, and potential immunogenicity of lung matrix, need to be resolved. To advance these various areas, in vitro, and in vivo work in large and small animal models will continue to be required. But beyond the application of natural scaffolds and stem cells in tissue engineering, these ex vivo culture systems will facilitate studies of both basic pathology and lung development and will assist the development of novel therapies going forward.

\section{AUTHOR CONTRIBUTIONS}

L.E.N. and M.G. designed the research and wrote the manuscript; M.G. generated the iPSC-derived epithelial cells and performed most the analyses; A.V.L., K.R., J.J.M., R.N.D., and P.B. performed lung decellularization, cell culture, and cell seeding into engineered lungs. G.H. and A.B. performed animal surgery, A.S. and A.V.L. analysed the engineered tissues. E.S.W. helped with study design.

\section{ACKNOWLEDGEMENTS}

We gratefully acknowledge Prof. James Thomson for providing human iPSCs clones. Human airway cells were provided by Dr. Randell under the auspices of NIH DK65988. Decellularized human lung were provided by Dr. White under the funds from National Institute of Health (NIH) U01 HL111016 for the U01 and the Drews Sarcoidosis Research Fund. This work was supported by NIH U01 HL111016 and R01 HL098220 (both to L.E.N.). This work was also supported by 14-SCA-YALE-18 from CT Stem Cell Innovation and NIH U01HI10967 (Duke Award number: 203-8675; both to M.G.). J.J.M., A.L., and A.T. were supported by T32 training grant no. 1T32GM086287. K.L.L was supported by the Medical Scientist Training Program, NIH/NIGMS T32 GM007205.

\section{CONFLICT OF INTEREST}

L.E.N. has a founder and shareholder in Humacyte, Inc., which is a regenerative medicine company. Humacyte produces engineered blood vessels from allogeneic smooth muscle cells for vascular surgery. L.E.N.'s spouse has equity in Humacyte, and L.E.N. serves on Humacyte's Board of Directors. L.E.N. is an inventor on patents that are licensed to Humacyte and that produce royalties for L.E.N. L.E.N. has received an unrestricted research gift to support research in her laboratory at Yale. Humacyte did not fund these studies, and 
Humacyte did not influence the conduct, description, or interpretation of the findings in this report.

\section{ORCID}

Mahboobe Ghaedi (i) http://orcid.org/0000-0001-9177-8739

Andrew V. Le (iD http://orcid.org/0000-0002-9301-0616

\section{REFERENCES}

Ali, N. N., Edgar, A. J., Samadikuchaksaraei, A., Timson, C. M., Romanska, H. M., Polak, J. M., \& Bishop, A. E. (2002). Derivation of type II alveolar epithelial cells from murine embryonic stem cells. Tissue Engineering, 8(4), 541-550.

Badylak, S. F., Weiss, D. J., Caplan, A., \& Macchiarini, P. (2012). Engineered whole organs and complex tissues. Lancet, 379(9819), 943-952.

Balestrini, J. L., Gard, A. L., Liu, A., Leiby, K. L., Schwan, J., Kunkemoeller, B., ... Niklason, L. E. (2015). Production of decellularized porcine lung scaffolds for use in tissue engineering. Integrative Biology (Camb), 7(12), 1598-1610. https://doi.org/10.1039/c5ib00063g

Beers, M. F., Moodley, Y., \& group RSCW (2017). When is an alveolar type 2 cell an alveolar type 2 cell? A conundrum for lung stem cell biology and regenerative medicine. American Journal of Respiratory Cell and Molecular Biology. https://doi.org/10.1165/rcmb.2016-0426PS. [Epub ahead of print]

Booth, A. J., Hadley, R., Cornett, A. M., Dreffs, A. A., Matthes, S. A., Tsui, J. L., ... White, E. S. (2012). Acellular normal and fibrotic human lung matrices as a culture system for in vitro investigation. American Journal of Respiratory and Critical Care Medicine, 186(9), 866-876.

Brouwer, K. M., Hoogenkamp, H. R., Daamen, W. F., \& van Kuppevelt, T. H. (2013). Regenerative medicine for the respiratory system: Distant future or tomorrow's treatment? American Journal of Respiratory and Critical Care Medicine, 187(5), 468-475.

D'Amour, K. A., Agulnick, A. D., Eliazer, S., Kelly, O. G., Kroon, E., \& Baetge, E. E. (2005). Efficient differentiation of human embryonic stem cells to definitive endoderm. Nature Biotechnology, 23(12), 1534-1541.

Duan, Y., Ma, X., Zou, W., Wang, C., Bahbahan, I. S., Ahuja, T. P., ... Zern, M. A. (2010). Differentiation and characterization of metabolically functioning hepatocytes from human embryonic stem cells. Stem Cells, 28(4), 674-686.

Fulcher, M. L., \& Randell, S. H. (2013). Human nasal and tracheo-bronchial respiratory epithelial cell culture. Methods in Molecular Biology, 945 109-121.

Garcia, O., Carraro, G., Navarro, S., Bertoncello, I., McQualter, J., Driscoll, B., ... Warburton, D. (2012). Cell-based therapies for lung disease. British Medical Bulletin, 101, 147-161.

Ghaedi, M., Calle, E. A., Mendez, J. J., Gard, A. L., Balestrini, J., Booth, A., ... Niklason, L. E. (2013). Human iPS cell-derived alveolar epithelium repopulates lung extracellular matrix. Journal of Clinical Investigation, 123(11), 4950-4962. https://doi.org/10.1172/JCl68793

Gilpin, S. E., Charest, J. M., Ren, X., \& Ott, H. C. (2016). Bioengineering lungs for transplantation. Thoracic Surgery Clinics, 26(2), 163-171. https://doi.org/10.1016/j.thorsurg.2015.12.004

Gilpin, S. E., Charest, J. M., Ren, X., Tapias, L. F., Wu, T., Evangelista-Leite, D., ... Ott, H. C. (2016). Regenerative potential of human airway stem cells in lung epithelial engineering. Biomaterials, 108, 111-119.

Gilpin, S. E., Ren, X., Okamoto, T., Guyette, J. P., Mou, H., Rajagopal, J., ... Ott, H. C. (2014). Enhanced lung epithelial specification of human induced pluripotent stem cells on decellularized lung matrix. The Annals of Thoracic Surgery, 98(5), 1721-1729.

Gomperts, B. N., \& Strieter, R. M. (2007). Stem cells and chronic lung disease. Annual Review of Medicine, 58, 285-298.

Green, M. D., Chen, A., Nostro, M. C., d'Souza, S. L., Schaniel, C., Lemischka, I. R., ... Snoeck, H. W. (2011). Generation of anterior foregut endoderm from human embryonic and induced pluripotent stem cells. Nature Biotechnology, 29(3), 267-272.
Hashimoto, S., Chen, H., Que, J., Brockway, B. L., Drake, J. A., Snyder, J. C., ... Stripp, B. R. (2012). $\beta$-Catenin-SOX2 signaling regulates the fate of developing airway epithelium. Journal of Cell Science 15;, 125(Pt 4), 932-942.

Huang, S. X., Islam, M. N., O'Neill, J., Hu, Z., Yang, Y. G., Chen, Y. W., ... Snoeck, H. W. (2014). Efficient generation of lung and airway epithelial cells from human pluripotent stem cells. Nature Biotechnology, 32(1), 84-91.

Huang, S. X. L., Islam, M. N., O'Neill, J., Hu, Z., Yang, Y. G., Chen, Y. W., ... Snoeck, H. W. (2013). Highly efficient generation of airway and lung epithelial cells from human pluripotent stem cells.

Kotton, D. N. (2012). Next-generation regeneration: The hope and hype of lung stem cell research. American Journal of Respiratory and Critical Care Medicine, 185(12), 1255-1260.

Kubo, A., Shinozaki, K., Shannon, J. M., Kouskoff, V., Kennedy, M., Woo, S., ... Keller, G. (2004). Development of definitive endoderm from embryonic stem cells in culture. Development, 131(7), 1651-1662.

Kubo, H. (2012). Tissue engineering for pulmonary diseases: Insights from the laboratory. Respirology, 17(3), 445-454.

Longmire, T. A., Ikonomou, L., Hawkins, F., Christodoulou, C., Cao, Y., Jean, J. C., ... Kotton, D. N. (2012). Efficient derivation of purified lung and thyroid progenitors from embryonic stem cells. Cell Stem Cell, 10(4), 398-411.

McCurry, K. R., Shearon, T. H., Edwards, L. B., Chan, K. M., Sweet, S. C., Valapour, M., ... Murray, S. (2009). Lung transplantation in the United States1998-2007. American Journal of Transplantation, 9(Part 2), 942-958.

Mou, H., Zhao, R., Sherwood, R., Ahfeldt, T., Lapey, A., Wain, J., ... Rajagopal, J. (2012). Generation of multipotent lung and airway progenitors from mouse ESCs and patient-specific cystic fibrosis iPSCs. Cell Stem Cell, 10(4), 385-397.

Nishikawa, S., Goldstein, R. A., \& Nierras, C. R. (2008). The promise of human induced pluripotent stem cells for research and therapy. Nature Reviews. Molecular Cell Biology, 9(9), 725-729.

Ott, H. C., Clippinger, B., Conrad, C., Schuetz, C., Pomerantseva, I., Ikonomou, L., ... Vacanti, J. P. (2010). Regeneration and orthotopic transplantation of a bioartificial lung. Nature Medicine, 16(8), 927-933.

Ott, H. C., \& Mathisen, D. J. (2011). Bioartificial tissues and organs: Are we ready to translate? Lancet, 378(9808), 1977-1978.

Petersen, T. H., Calle, E. A., Colehour, M. B., \& Niklason, L. E. (2011). Bioreactor for the long-term culture of lung tissue. Cell Transplantation, 20(7), 1117-1126.

Petersen, T. H., Calle, E. A., \& Niklason, L. E. (2011). Strategies for lung regeneration. Materials Today, 14(5), 196-201.

Petersen, T. H., Calle, E. A., Zhao, L., Lee, E. J., Gui, L., Raredon, M. B., ... Niklason, L. E. (2010). Tissue-engineered lungs for in vivo implantation. Science, 329(5991), 538-541.

Price, A. P., England, K. A., Matson, A. M., Blazar, B. R., \& PanoskaltsisMortari, A. (2010). Development of a decellularized lung bioreactor system for bioengineering the lung: The matrix reloaded. Tissue Engineering. Part A, 16(8), 2581-2591.

Rackley, C. R., \& Stripp, B. R. (2012). Building and maintaining the epithelium of the lung. The Journal of Clinical Investigation, 122(8), 2724-2730.

Rana, P., Anson, B., Engle, S., \& Will, Y. (2012). Characterization of humaninduced pluripotent stem cell-derived cardiomyocytes: Bioenergetics and utilization in safety screening. Toxicological Sciences, 130(1), 117-131.

Rawlins, E. L., \& Hogan, B. L. (2006). Epithelial stem cells of the lung: Privileged few or opportunities for many? Development, 133(13), 2455-2465.

Rock, J. R., Gao, X., Xue, Y., Randell, S. H., Kong, Y. Y., \& Hogan, B. L. (2011). Notch-dependent differentiation of adult airway basal stem cells. Cell Stem Cell 3; 8(6), 639-648.

Song, J. J., \& Ott, H. C. (2011). Organ engineering based on decellularized matrix scaffolds. Trends in Molecular Medicine, 17(8), 424-432. 
Takahashi, K., Tanabe, K., Ohnuki, M., Narita, M., Ichisaka, T., Tomoda, K., \& Yamanaka, S. (2007). Induction of pluripotent stem cells from adult human fibroblasts by defined factors. Cell Proliferation, 131(5), 861-872.

Tesei, A., Zoli, W., Arienti, C., Storci, G., Granato, A. M., Pasquinelli, G., ... Bonafè, M. (2009). Isolation of stem/progenitor cells from normal lung tissue of adult humans. Cell Proliferation, 42(3), 298-308.

Wagner, D. E., Bonvillain, R. W., Jensen, T., Girard, E. D., Bunnell, B. A., Finck, C. M., ... Weiss, D. J. (2013). Can stem cells be used to generate new lungs? Ex vivo lung bioengineering with decellularized whole lung scaffolds. Respirology, 18(6), 895-911.

Wang, D., Haviland, D. L., Burns, A. R., Zsigmond, E., \& Wetsel, R. A. (2007). A pure population of lung alveolar epithelial type II cells derived from human embryonic stem cells. Proceedings of the National Academy of Sciences of the United States of America, 104(11), 4449-4454.

Weiss, D. J., Bates, J. H., Gilbert, T., Kim, C., Panoskaltsis-Mortari, A., Reynolds, S., ... Prockop, D. J. (2013). Conference report: Stem cells and cell therapies in lung biology and diseases University of Vermont. Annals of the American Thoracic Society, 10(5), S25-S44. https://doi. org/10.1513/AnnalsATS.201304-089AW

Wong, A. P., Bear, C. E., Chin, S., Pasceri, P., Thompson, T. O., Huan, L. J., ... Rossant, J. (2012). Directed differentiation of human pluripotent stem cells into mature airway epithelia expressing functional CFTR protein. Nature Biotechnology, 30(9), 876-882.

Yu, J., Vodyanik, M. A., Smuga-Otto, K., Antosiewicz-Bourget, J., Frane, J. L., Tian, S., ... Thomson, J. A. (2007). Induced pluripotent stem cell lines derived from human somatic cells. Science, 318(5858), 1917-1920.

Zorn, A. M., \& Wells, J. M. (2009). Vertebrate endoderm development and organ formation. Annual Review of Cell and Developmental Biology, 25, 221-225.221. https://doi.org/10.1146/annurev.cellbio.042308.113344

\section{SUPPORTING INFORMATION}

Additional Supporting Information may be found online in the supporting information tab for this article.

Data S1. This article has Supplemental Methods, 2 Supplementary Tables and 7 Supplementary Figures.

Table S1. List of antibodies used in staining, flow cytometry and western blot for various experiments.

Table S2. Sequences of primers used in qRT-PCR for various experiments.

Supplementary text - Additional information regarding the Chemical and Reagents, protocol for iPSC differentiation to lung epithelial cells, and protocols for decellularization and recellularization of rat, and human lung.

Supplementary tables - provide the list of primary and secondary antibodies used in staining, and primers sequence for various experiments in this study.

How to cite this article: Ghaedi M, Le AV, Hatachi G, et al. Bioengineered lungs generated from human iPSCs-derived epithelial cells on native extracellular matrix. J Tissue Eng Regen Med. 2018;12:e1623-e1635. https://doi.org/10.1002/ term.2589 